\title{
Loudness Adaptation in Acoustic and Electric Hearing
}

\author{
Qing TAnG, ${ }^{1,2,3,4}$ Sheng Liu, ${ }^{1,2,3}$ And Fan-Gang ZenG ${ }^{1,2,3,4}$ \\ ${ }^{1}$ Department of Anatomy and Neurobiology, University of California, Irvine, CA 92697, USA \\ ${ }^{2}$ Department of Biomedical Engineering, University of California, Irvine, CA 92697, USA \\ ${ }^{3}$ Department of Cognitive Sciences and Otolaryngology-Head and Neck Surgery, University of California, Irvine, \\ CA 92697, USA \\ ${ }^{4}$ Hearing and Speech Research Laboratory, University of California, 364 Med Surge II, Irvine, CA 92697-1275, USA
}

Received: 31 May 2005; Accepted: 29 November 2005; Online publication: 20 January 2006

\begin{abstract}
The present study is aimed to evaluate and compare loudness adaptation between normal hearing and cochlear-implant subjects. Loudness adaptation for 367 -s pure tones was measured in five normal-hearing subjects at three frequencies $(125,1,000$, and 8,000 $\mathrm{Hz})$ and three levels $(30,60$, and $90 \mathrm{~dB}$ SPL). In addition, loudness adaptation for 367-s pulse trains was measured in five Clarion cochlear-implant subjects at three stimulation rates $(100,991$, and 4,296 $\mathrm{Hz})$, three levels $(10,50$, and $90 \%$ of the electric dynamic range), three stimulation positions (apical, middle and basal), and two stimulation modes (monopolar and bipolar). The method of successive magnitude estimation was used to quantify loudness adaptation. Similar to the previous results, we found that loudness adaptation in normal-hearing subjects increases with decreasing level and increasing frequency. However, we also found a small but significant loudness enhancement at $90 \mathrm{~dB}$ SPL in acoustic hearing. Despite large individual variability, we found that loudness adaptation in cochlear-implant subjects increases with decreasing levels, but is not significantly affected by the rate, place and mode of stimulation. A phenomenological model was proposed to predict loudness adaptation as a function of stimulus frequency and level in acoustic hearing. The present results were not fully compatible with
\end{abstract}

Correspondence to: Qing Tang and Fan-Gang Zeng - Hearing and Speech Research Laboratory - University of California • 364 Med Surge 1l, Irvine, CA 92697-1275, USA. Telephone: 949-824-1539; fax: 949-824-5907; email: fzeng@uci.edu; qtang@uci.edu either the restricted excitation hypothesis or the neural adaptation hypothesis. Loudness adaptation may have a central component that is dependent on the peripheral excitation pattern.

Keywords: cochlear implant, loudness, loudness adaptation, stimulation mode

\section{INTRODUCTION}

Loudness is a subjective attribute of sound intensity, but can also be significantly affected by stimulus frequency and duration (ISO 1966). Keeping other stimulus parameters constant, loudness is a power function of sound intensity in acoustic hearing, but an exponential function in electric hearing (Stevens 1970; Zeng and Shannon 1994).

Loudness adaptation is defined as a decrease in loudness for a sustained, fixed-level tone. Loudness adaptation is typically measured by a comparison tone presented either in the opposite ear at the same time or in the same ear right after the test tone (Hood 1950). Previous studies found that loudness adaptation depends on both sound level and frequency. Over the course of several minutes, the loudness of a sustained, fixed-level pure-tone can decrease by $70-100 \%$ at $5 \mathrm{~dB}$ sensation level (SL), $20 \%$ at $40 \mathrm{~dB}$ SL, and stay essentially unchanged at higher SLs (Hellman et al. 1997). Loudness adaptation occurs mostly with high-frequency sounds $(>4,000 \mathrm{~Hz})$ while low-frequency sounds show little or no adaptation (Miskiewicz et al. 1993; Hellman et al. 1997). 
Loudness adaptation has been used as a diagnostic tool to differentiate between hearing loss of the cochlear or retro-cochlear origin (Hood 1955; Carhart 1957). Tone decay, or total loudness adaptation, is a classic example. In hearing-impaired subjects with retro-cochlear lesions, such as neural degeneration, inflammation, trauma and space-occupying lesions, a sound with a constant level may become totally inaudible with time (Carhart 1957; Green 1985). Total loudness adaptation rarely occurs in normal-hearing and cochlear-impaired listeners.

Although the physiological mechanisms underlying loudness growth are not clear, they have been related to both cochlear and central nonlinearities (Fowler 1936; Zeng and Shannon 1994; Schlauch et al. 1998; Schlauch et al. 2001), as well as the total number and the timing of neural discharges in the auditory system (Fletcher 1940; Robinson and Dadson 1956; Carney 1994; Moore 1995; Relkin and Doucet 1997). Quantitative loudness models in acoustic and electric hearing have been proposed based on physiological data from the cochlear spread of excitation patterns and the auditory nerve activities (Zwicker and Scharf 1965; Bruce et al. 1999a, b; Moore and Glasberg 2004).

The mechanisms underlying loudness adaptation are also unclear. One possible mechanism suggests that loudness adaptation is caused by the "restricted excitation pattern" in the cochlea (Scharf 1983). The restricted excitation mechanism can explain the observed frequency and level effects on adaptation. Both high-frequency and low-level tones produce more restricted excitation, and accordingly more adaptation than low-frequency and high-level tones (Scharf 1983). Quantitatively, the restricted excitation mechanism further suggests that the spread of excitation towards the basal part be used to calculate the amount of loudness adaptation (Miskiewicz et al. 1993).

An alternative mechanism directly relates loudness adaptation to long-term adaptation properties at the auditory nerve level (Winter et al. 1990; Javel 1996). Javel observed that driven responses of cat auditory nerve fibers to constant stimuli decrease substantially over time periods ranging from seconds to minutes. The long-term neural adaptation increased with SL and decreased with spontaneous activity, but was not significantly correlated with the fiber's characteristic frequency or threshold. On average, the amount of decrease in discharge rate was about $40 \%$ and the time constant was 3-4 s in high-spontaneous-rate fibers for stimuli presented at 20-40 dB SL. Javel noticed that these values obtained in cat auditory nerve fibers closely resembled that observed in human loudness adaptation (Miskiewicz et al. 1993), suggesting a peripherally based neural mechanism for loudness adaptation. One problem with the neural adaptation mechanism is that neural adaptation increases with increasing stimulation level but loudness adaptation decreases with increasing stimulation level. It is possible that loudness adaptation has a central component, depending upon the number, distribution and timing of nerve spikes across characteristic frequencies (Huss and Moore 2003).

Because the cochlear implant bypasses the damaged inner ear and directly stimulates the auditory nerve fibers, we expected that comparing loudness adaptation between acoustic and electric hearing would also provide a unique opportunity to assess the relative contribution of peripheral and central mechanisms to loudness adaptation. However, systematic studies of loudness adaptation in electric hearing are lacking. Several prior studies used Carhart's method (Carhart 1957) to measure tone decay in electric hearing (Brimacombe and Eisenberg 1984; Sennaroglu et al. 2001; Wable et al. 2001). While Carhart's method provided valuable clinical information on tone decay, it did not accurately quantify loudness adaptation in terms of the amount and the time course of loudness adaptation.

Recently, a high-rate conditioner has been used to restore spontaneous activities (Rubinstein et al. 1999) and to suppress tinnitus (Rubinstein et al. 2003) in cochlear implant users. Potential benefits of the highrate stimulation include an increased electric dynamic range, and improved encoding of temporal fine structure and speech perception (Wilson et al. 1993; Pelizzone 1998; Hong et al. 2003). While the highrate conditioner has been reported to produce total adaptation within several minutes of stimulation, systematic measurement is lacking (Hong et al. 2003).

The present study systematically measured loudness adaptation in acoustic and electric hearing to achieve the following two goals. First, the present study replicated previous studies in acoustic hearing and expanded on them by examining the influence of high-level sounds (90 dB SPL) on loudness adaptation. We also developed a quantitative model of loudness adaptation in acoustic hearing. Second, the present study systematically and comprehensively examined the effects of stimulation rate, level, place, and mode on loudness adaptation. The electric loudness adaptation data allowed us to explicitly test several working hypotheses that were difficult to test in acoustic hearing. The restricted excitation mechanism predicted greater degrees of loudness adaptation with basal electrodes than apical electrodes, and additionally with the bipolar mode than the monopolar mode. The neural adaptation model predicted that loudness adaptation is independent of electrode position and stimulation mode, and that it increases with increasing stimulate rate and level. 


\section{EXPERIMENT 1: ACOUSTIC HEARING}

Materials and methods

Subjects. Five normal-hearing subjects, three females and two males, from ages 22 to 27 years old, participated in Experiment 1. All subjects had normal pure-tone audiograms at octave frequencies from 125 to $8,000 \mathrm{~Hz}$ and subjectively reported as having heard all of the test tones, including the 125$\mathrm{Hz}, 30-\mathrm{dB}$ SPL sound. They were compensated for their participation. The local Institute Review Board approved the research protocol and informed consent before human subjects testing began.

Stimuli. All stimuli were generated digitally using the Tucker-Davis Technologies (TDT) system III (Gainesville, FL). A 16-bit D/A converter was used with a 44,100-Hz sampling rate. A 2.5-ms ramp was applied to all stimuli to avoid spectral splatter. To calibrate the sound acoustically, the full digital range was used to generate a $1,000-\mathrm{Hz}$ tone that produced $100 \mathrm{~dB}$ SPL in Sennheiser HAD200 headphones (Wedemark, Germany), measured by a B\&K Type 2260 sound level meter in a Zwislocki real-ear stimulator (Narum, Denmark). The corresponding voltage $(132 \mathrm{mV})$ was monitored daily by a multimeter, whereas acoustic calibration was conducted periodically for overall equipment calibration and maintenance.

Test stimuli were 367 -s pure tones at three frequencies $(125,1,000$ and $8,000 \mathrm{~Hz}$ ) and three levels (30, 60 and $90 \mathrm{~dB}$ SPL). The subject performed all tasks in front of a computer terminal while sitting inside a nine by ten squared-foot, double-walled, sound-insulated booth (Industrial Acoustics Co. Bronx, NY).

Procedure. To familiarize the subjects with the range of sounds to be presented, all stimulus combinations were presented to subjects using a 3-s duration and a 3-s interval. Doing so would also likely produce more reliable measurement of loudness adaptation using the successive magnitude estimation method. Loudness adaptation of pure tones at different frequencies and levels was then tested one by one. Before the onset of each continuous sound, the listeners heard two 500-ms tone bursts with the same level and frequency as the continuous sound to alert them of the coming of the continuous sound.

The method of successive magnitude estimation was used to measure loudness adaptation, in which a number was assigned to the perceived loudness at any given moment of observation (Scharf 1983). The judgment was made without any designated standard. Instead, listeners were free to use any positive number, including decimals, regardless of the number they assigned to the previous stimulus. Zero was used to indicate that the sound was inaudible.
Hellman et al. (1997) compared the reliability of the successive magnitude estimation with that of a loudness matching method, in which the listener matched the continuous tone by using a 1-s tone burst presented intermittently in the opposite ear and found similar results between these two methods (Hellman et al. 1997).

At 2-s after the onset of the 367-s continuous tone, and each 20-s thereafter, which was indicated by a visual signal on the computer screen, the subject had to assign a number to the loudness of the tone at that moment within a 15-s window after the visual signal. The listener judged the loudness for a total of 19 measurements for each frequency-level combination (Hellman et al. 1997). One trial for each of the nine frequency-level combinations was presented to the listener. The order of trials was randomized with the exception that two trials with the same frequency could not occur in succession. This constraint was intended to minimize residual adaptation that might be introduced by the same frequency. Residual effects proved to be large when an exposure tone was presented twice in a row at the same frequency after a pause of less than approximately $6 \mathrm{~min}$ (Miskiewicz and Hellman 1995). In this experiment, the minimum pause between test sessions was increased to $8 \mathrm{~min}$. All of the cases were divided into two test sessions, each lasting about $1 \mathrm{~h}$.

Analysis. In order to measure the amount of change in loudness perception during the test time course, the following equation was used to transform the successive magnitude estimates for each listener to the percentage of loudness adaptation:

$$
\begin{aligned}
& \text { Loudness adaptation percentage } \\
& \quad=100 *\left(M_{t}-M_{0}\right) / M_{0}
\end{aligned}
$$

where $M_{0}$ is the first loudness estimate at 2-s after the onset of the continuous sound, $M_{t}$ is the loudness estimate at time $t$. A value of $-100 \%$ means that the sound is inaudible $\left(M_{t}=0\right)$, zero means no adaptation $\left(M_{t}=M_{0}\right)$, and a positive value means loudness enhancement. In order to make it easier to visualize and understand the time course of the adaptation and the magnitude of the plateaued adaptation percentage, we fitted the adaptation data using the following equation:

$$
y(t)=s^{*}\left(1-e^{-t / \tau}\right)
$$

where $y(t)$ is the loudness adaptation percentage at time $t ; s$ stands for the plateaued adaptation percentage $(>260 \mathrm{~s})$, calculated as the average of the final five loudness estimates, in terms of percentage of the original loudness at the onset of stimulation. $s$ can be 


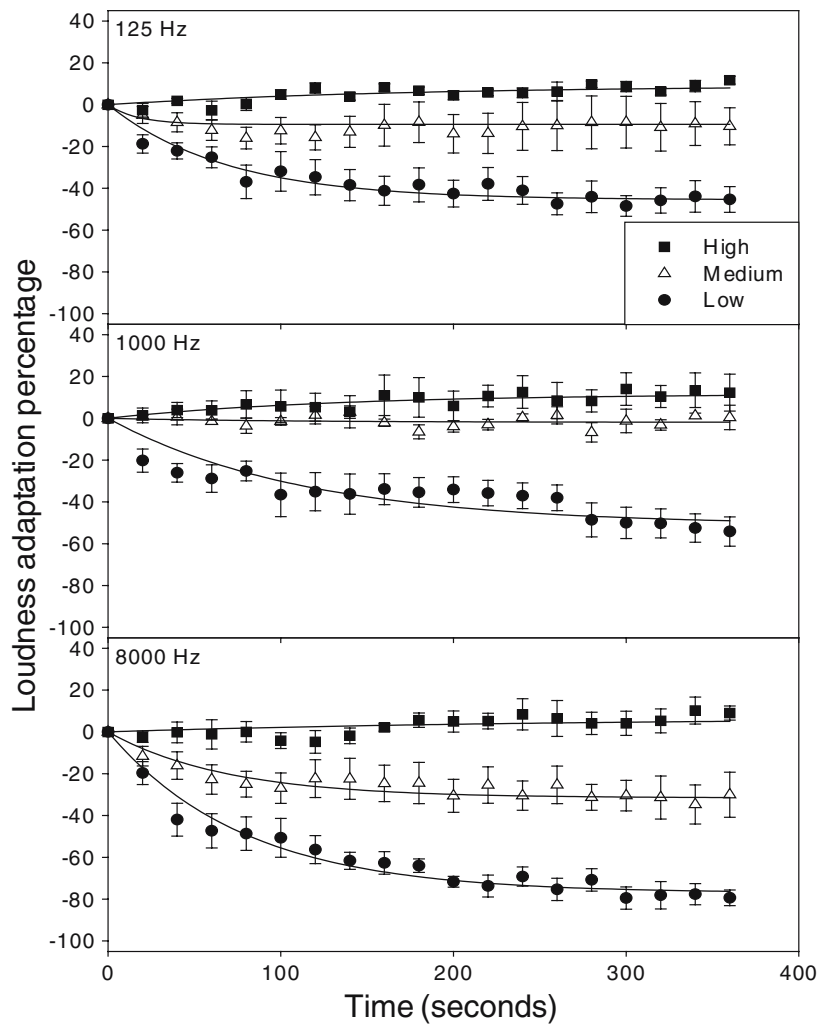

FIG. 1. Average percentage of loudness adaptation as a function of time in seconds after the onset of a continuous tone from five normal-hearing subjects. The vertical bars indicate \pm 1 standard error. The top, middle, and bottom panels represent stimulus frequency at 125, 1,000, and $8,000 \mathrm{~Hz}$, respectively. Circles, triangles, and squares represent stimulus level at 30,60, and $90 \mathrm{~dB}$ $\mathrm{SPL}$, respectively. The lines represent best fit to the data by Eq. 2 described in the main text.

negative, zero or a positive number, depending on the change of perceived loudness. $\tau$ is the time constant at which the loudness decreased or increased to $63.2 \%$ of the final saturation s. Eq. 2 was used to fit the data using SigmaPlot (Richmond, USA). In 41 out of 45 cases, the best fitting function could account for $80 \%$ or greater variance in the data (lower than $20 \%$ in the remaining four cases).

\section{Results}

Figure 1 shows the group mean data in percentages of loudness adaptation as a function of time at three stimulus frequencies (different panels) and three stimulus levels (different symbols). Error bars represent \pm 1 standard error of the mean. Regression lines are fitted to the data using Eq. 2 to derive both the plateaued adaptation percentage $(s)$ and the time constant $(\tau)$.

Two-way (level and frequency) repeated-measure ANOVA was used to analyze the data, showing that the plateaued adaptation percentage is significantly dependent upon stimulus level $[\mathrm{F}(2,8)=69.6, p<0.05]$ and stimulus frequency $[\mathrm{F}(2,8)=8.1, p<0.05]$. No significant interaction was found between stimulus level and frequency $[\mathrm{F}(4,16)=1.5, p>0.05]$. At the low stimulus level (30 dB SPL), all stimuli produced loudness adaptation, saturating at $-48.8,-51.1$, and $-77.0 \%$ for the $125-, 1,000-$, and $8,000-\mathrm{Hz}$ stimulus, respectively. At the high stimulus level (90 dB SPL), all stimuli produced loudness enhancement, saturating at $+9.1,+11.7$, and $+6.7 \%$ for the $125-, 1,000$-, and $8,000-\mathrm{Hz}$ stimulus, respectively. At the middle level (60 dB SPL), the two low-frequency stimuli produced essentially small or no adaptation $(-9.4$ and $-2.7 \%)$ while the high-frequency stimuli produced $-30.9 \%$ adaptation. On the other hand, no significant difference in time constant was found for stimulus level $[\mathrm{F}(2,8)=1.3, p>0.05]$ and frequency $[\mathrm{F}(2,8)=0.3$, $\mathrm{p}>0.05]$. On average, the time constant was $78 \mathrm{~s}$ for loudness adaptation and $90 \mathrm{~s}$ for loudness enhancement.

\section{EXPERIMENT 2: ELECTRIC HEARING}

Materials and methods

Subjects. Five post-lingually deafened Clarion-I cochlear-implant users participated in Experiment 2. Table 1 shows etiology, history, speech-processing strategy and stimulation mode in these subjects.

TABLE 1

Subject demographical information

\begin{tabular}{|c|c|c|c|c|c|c|}
\hline Subject & Age & Etiology & $\begin{array}{c}\text { Duration of } \\
\text { hearing loss (year) }\end{array}$ & $\begin{array}{l}\text { Duration of } \\
\text { Cl use (year) }\end{array}$ & Speech strategy & Electrode type \\
\hline $\mathrm{Cl} 1$ & 21 & Congenital & 21 & 4.25 & SAS & Hifocus bipolar \\
\hline $\mathrm{Cl} 2$ & 66 & Meniere's syndrome & 48 & 6 & SAS & Enhanced bipolar \\
\hline $\mathrm{Cl} 3$ & 59 & Meniere's syndrome & 19 & 6 & $\mathrm{CIS}$ & Monopolar \\
\hline $\mathrm{Cl} 4$ & 55 & Progressive hearing loss & 44 & 10 & $\mathrm{CIS}$ & Monopolar \\
\hline $\mathrm{Cl} 5$ & 48 & Nerve deafness & 38 & 7 & SAS & Enhanced bipolar \\
\hline
\end{tabular}

The duration of hearing loss was defined as the time at which the subject first noticed hearing loss to the time at which the subject received a cochlear implant. Speech strategies used include Simultaneous Analogue Stimulation (SAS) and Continuous Interleaved Sampling (CIS). 
Their ages ranged from 21 to 66 years old with a mean age of 50 years. The duration of implant use ranged from 4 to 10 years with a mean of 6 years and 8 months. Three speech processors of the five subjects were programmed with the Simultaneous Analogue Stimulation (SAS) and the other two were programmed with Continuous Interleaved Sampling (CIS) speech-processing strategies. The monopolar mode was used for the CIS users while the bipolar mode was used for the SAS users. Two subjects were using hearing aids in the unimplanted ear and were asked to turn them off during testing.

Stimuli. All electric stimuli were generated and controlled by a research interface provided by Advanced Bionics Corporation for the Clarion C-I Intracochlear Stimulator (ICS). The electrode array of Clarion C-I has 16 spherical contacts arranged in eight radial bipolar pairs. The electrode array was designed to hug the modiolar wall of the cochlea, presumably placing electrodes in close proximity to the spiral ganglion cells.

The stimuli were charge-balanced, biphasic pulses, with $232.8-\mu \mathrm{s} /$ phase at the $100-$ and $991-\mathrm{Hz}$ stimula- tion rate, or $77.6-\mu \mathrm{s} /$ phase at the $4,296-\mathrm{Hz}$ stimulation rate. The initial phase of the biphasic pulses delivered to the electrode was anodic. The duration of the stimuli was $500 \mathrm{~ms}$ for measuring the electric dynamic range (threshold and the maximal comfortable loudness level). The duration was $367 \mathrm{~s}$ for the loudness adaptation test. At each stimulation rate, three monopolar electrode configurations $[(1,0)$, $(4,0)$ and $(8,0)]$ and three bipolar configurations $[(1,2),(4,5)$ and $(7,8)]$, were used. In the monopolar mode, 0 represents the indifferent electrode band located on the case of the ICS, while one to eight represented the eight medial electrodes, arranged from apical to basal positions. The threshold and maximal comfortable level were measured for each rate and electrode configuration in each subject to produce the electric dynamic range data. Stimulation levels were set at 10,50 and $90 \%$ of the dynamic range to represent low, medium and high loudness levels for each specific stimulation rate, electrode position, and stimulation mode. Adaptation was measured for a total of 54 combinations (3-levels $\times 3$ rates $\times 3$-positions $\times 2$-modes). All subjects completed

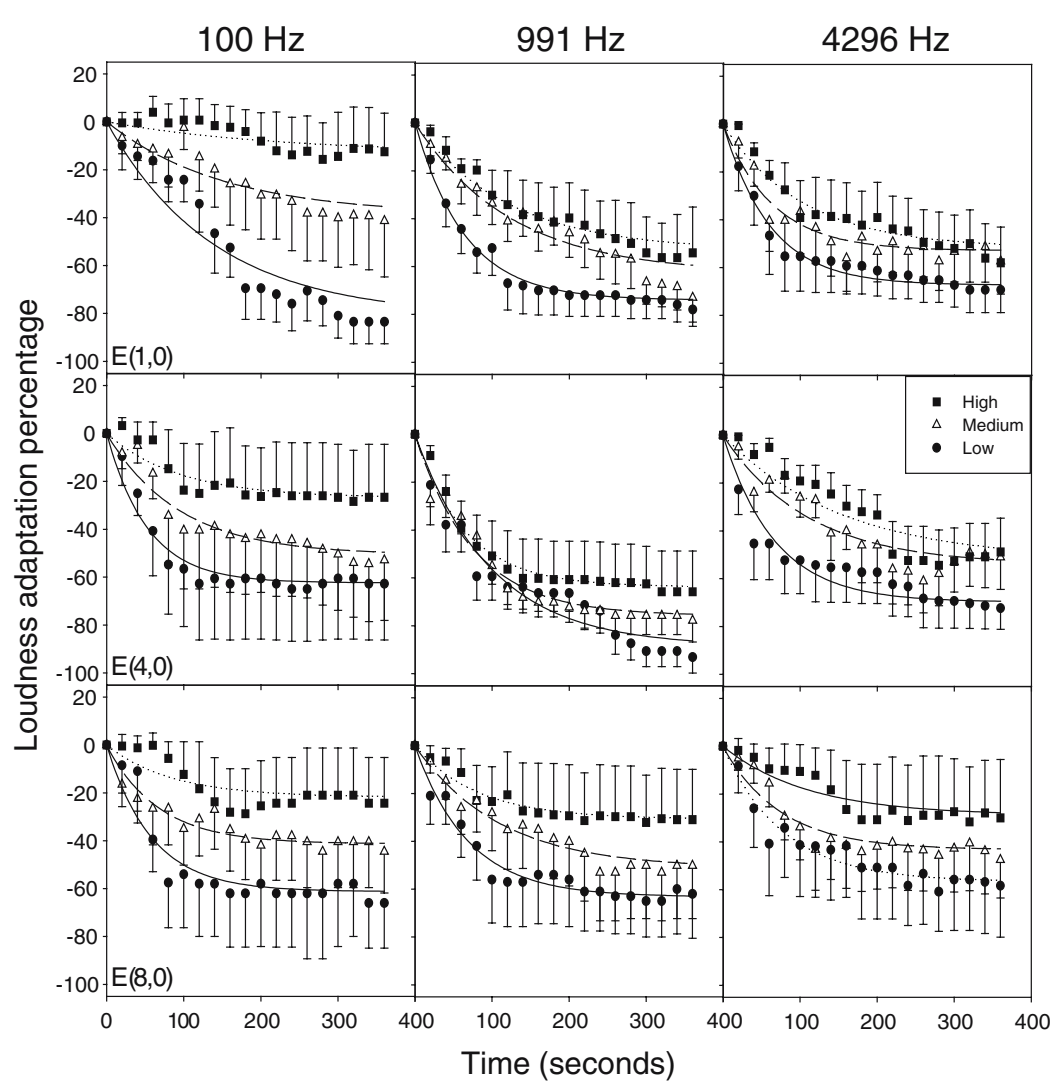

FIG. 2. Mean percentage of loudness adaptation for five cochlear implant subjects as a function of time in seconds after the onset of a pulse train stimuli. The vertical bars indicate \pm 1 standard error. The nine panels represent nine different combinations of stimulation frequency and stimulation electrode pairs. Within each panel, circles, triangles, and squares represent low, medium, and high stimulus levels, respectively. The lines show the linear regression fit by Eq. 2 in the main text. 


\section{TABLE 2}

Individual plateaued percentage $(s$ in $\%)$ /time constant $(\tau$ in seconds) in five Clarion-I subjects

Plateaued percentage (s)

Time constant $(\tau)$

$a_{0}=-116.248$

$a_{1}=2.3826$

$a_{2}=2.46366$

$a_{3}=-0.586328$

$a_{4}=-0.0112244$

$b_{0}=228.226$

$b_{1}=37.6673$

$b_{2}=-8.13462$

$b_{3}=-3.92351$

$b_{4}=0.0810201$

the 27-monopolar combinations, but three subjects could complete only part of the bipolar combinations due to the hardware limitation (requiring higher currents than the implant could reliably deliver).

Procedure. The threshold was determined by an adaptive, three-interval, two-alternative forced choice procedure (3IFC) with feedback. The signal level was varied according to a two-down, one-up decision rule to estimate the $71 \%$ correct point on the psychometric function (Green 1990). Level decreased with two right responses and increased with one wrong response. A reversal occurred with a change in the level direction (from increase to decrease or vice versa). The step size for the signal level change was large $(10 \mu \mathrm{A})$ for the first four reversals but small $(2.5$ $\mu \mathrm{A})$ for the next six to eight reversals. The threshold was estimated by averaging the values from the reversals with the small step size. After the threshold was determined, an ascending method was used to determine the maximal comfortable level (MCL) for each stimulation rate, electrode and stimulation mode. Only one run was conducted for threshold and MCL measures.

We used the same successive magnitude estimation as in the acoustic hearing experiment to measure loudness adaptation in electric hearing. A continuous pulse train of $367 \mathrm{~s}$ was presented to the implant. Two seconds after the onset of the stimulus, and every 20-s thereafter. The subject was asked to assign a number to the perceived loudness at the moment. The combinations were presented in random order with the exception that the same electrode pair could not occur in succession. The rest period between runs was 8 min.

Analysis. The same equations as in the acoustic hearing experiment were used to quantify the amount of loudness adaptation (Eq. 1) and to fit the loudness adaptation time course (Eq. 2). In two cases

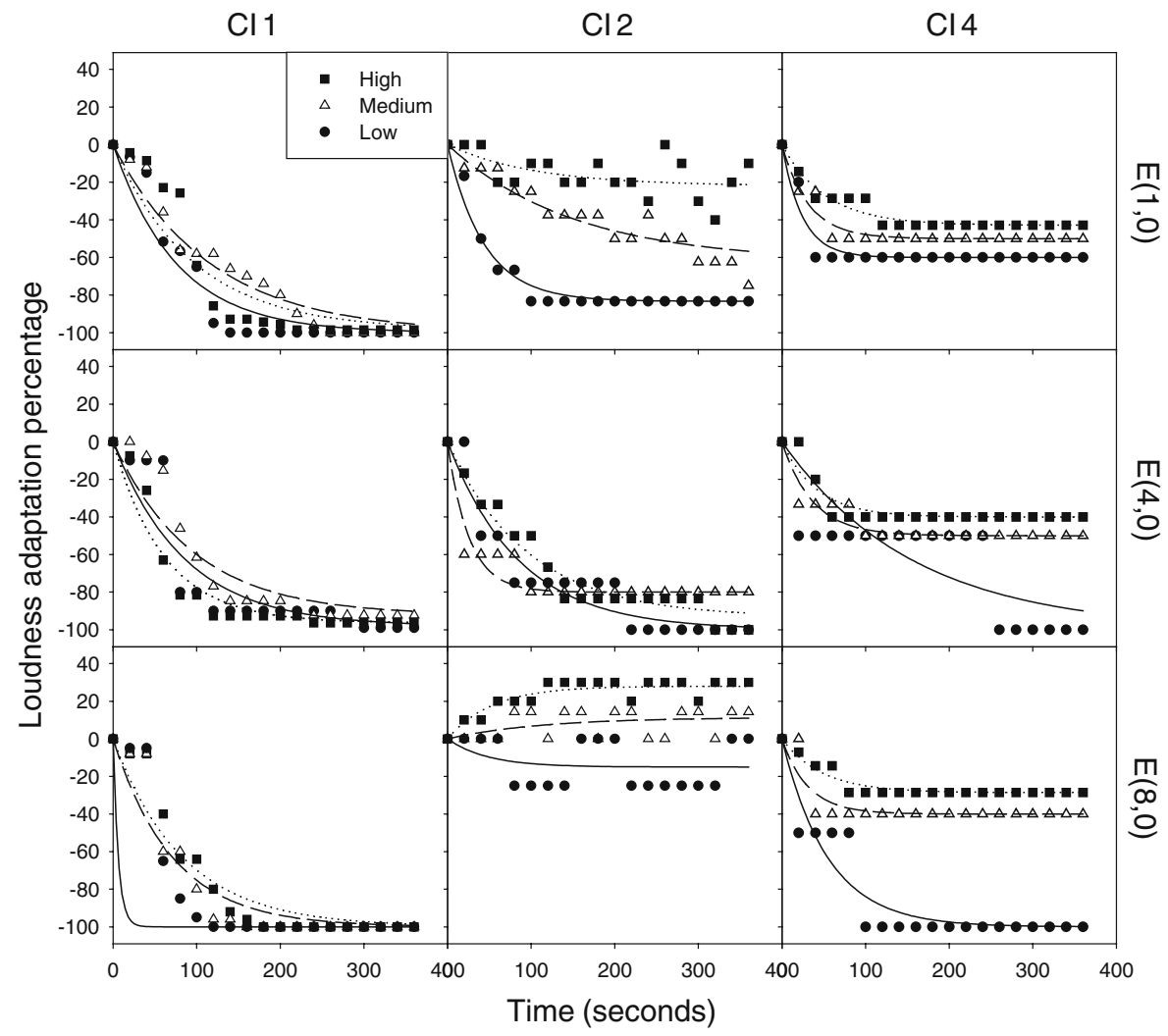

FIG. 3. Loudness adaptation for three cochlear implant subjects as a function of time in seconds at stimulation rate $991 \mathrm{~Hz}$. Three columns show data from $\mathrm{Cl}-1, \mathrm{Cl}-2$ and $\mathrm{Cl}-4$, respectively. The three rows show the electrode pairs $(1,0),(4,0)$ and $(8,0)$, respectively.
Within each panel, circles, triangles, and squares represent low, medium, and high stimulus levels, respectively. The lines show the linear regression fit by Eq. 2 in the main text. 


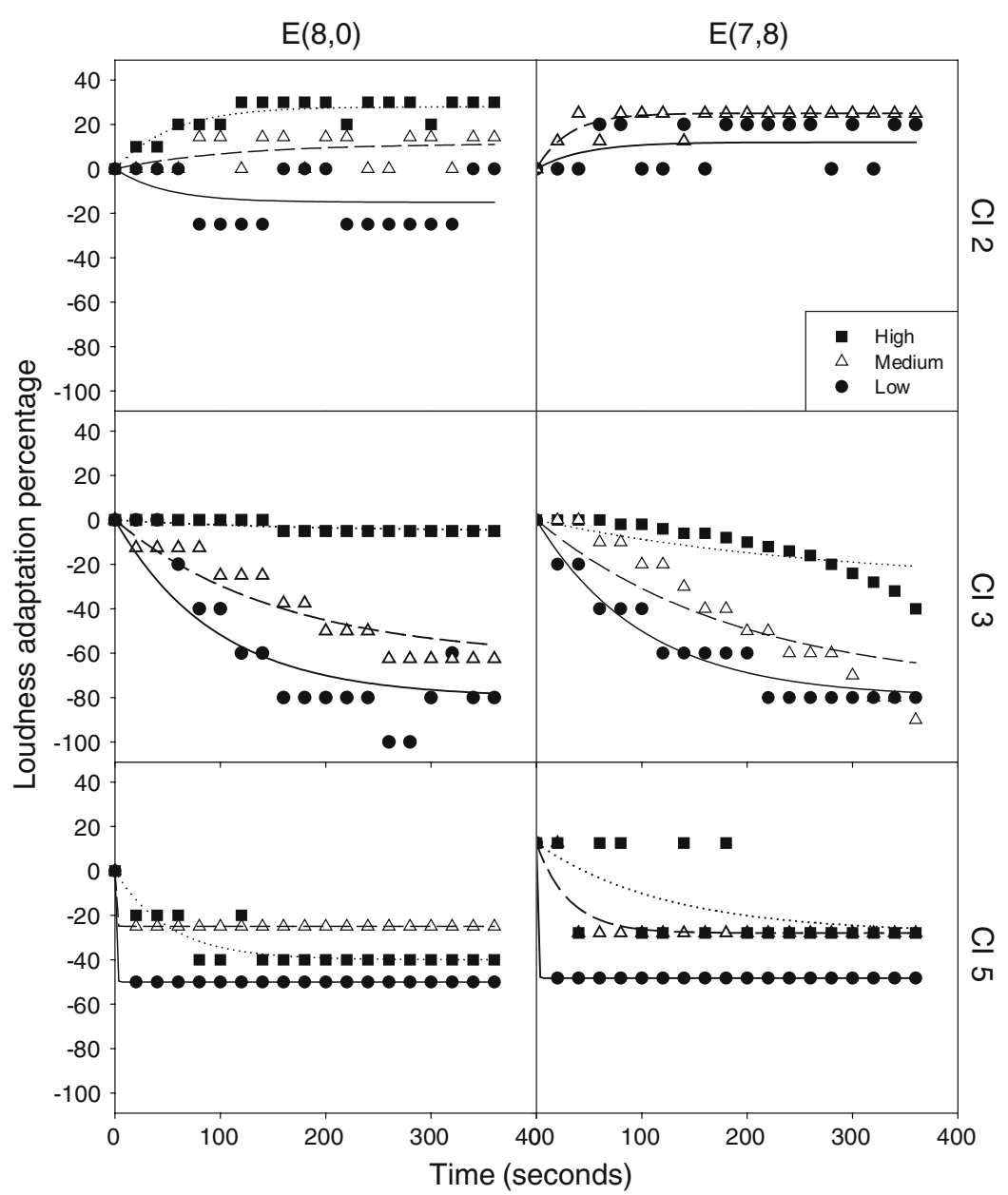

FIG. 4. Loudness adaptation comparison between monopolar and bipolar stimulation mode as a function of time in seconds at stimulation rate of $991 \mathrm{~Hz}$. The left column represents the monopolar stimulation mode at electrode pair $(8,0)$ and the right column represents the bipolar stimulation mode at electrode pair
$(7,8) . \mathrm{Cl}-2, \mathrm{Cl}-3$ and $\mathrm{Cl}-5$ are shown in three rows. Within each panel, circles, triangles, and squares represent low, medium, and high stimulus levels, respectively. The lines show the linear regression fit by Eq. 2 . for subject CI 2, the fitting failed because an oscillating pattern occurred with the perceived loudness. In these cases, 0 was used to indicate the plateaued adaptation percentage $(s)$ and time constant $(\tau)$.

Results. Figure 2 shows the group mean data in loudness adaptation as a function of time at different levels (different symbol), different stimulation rates (different columns) and different electrode pairs (different rows) with the monopolar mode. Error bars represent a standard error of the mean. Regression lines are fitted to the data using Eq. 2 to derive both the plateaued adaptation percentage $(s)$ and the time constant $(\tau)$.

A three-way (stimulus level, rate, and place), repeated-measure ANOVA was used to analyze the data, showing a significant effect of level $[F(2,8)=7.2$, $p<0.05]$, but not of rate $[\mathrm{F}(2,8)=3.5, p>0.05]$ and place $[\mathrm{F}(2,8)=1.2, p>0.05]$ on plateaued adaptation percentage. The time constant was found to be $99 \mathrm{~s}$, independent of stimulus level $[\mathrm{F}(2,8)=1.7, p>0.05]$, rate $[\mathrm{F}(2,8)=1.2, p>0.05]$, and place $[\mathrm{F}(2,8)=4.2$, $p>0.05]$. While the group mean data in electric hearing resembled that in acoustic hearing, greater individual variability was apparent in electric hearing (Table 2).

Table 2 shows complete individual data on plateaued adaptation percentage $(s)$ and time constant $(\tau)$ in all five implant subjects and all 27 conditions (3 rates $\times 3$ electrode place $\times 3$ levels $)$. We observed total loudness adaptation (*) $22 \%$ of the time, mostly in subject CI-1, loudness enhancement (**) $9 \%$ of the time, mostly in subject CI-2, and no loudness adaptation $(* * *) 4 \%$ of the time. To further demonstrate the individual data patterns, Fig. 3 shows two special cases and one typical case in electric hearing. 


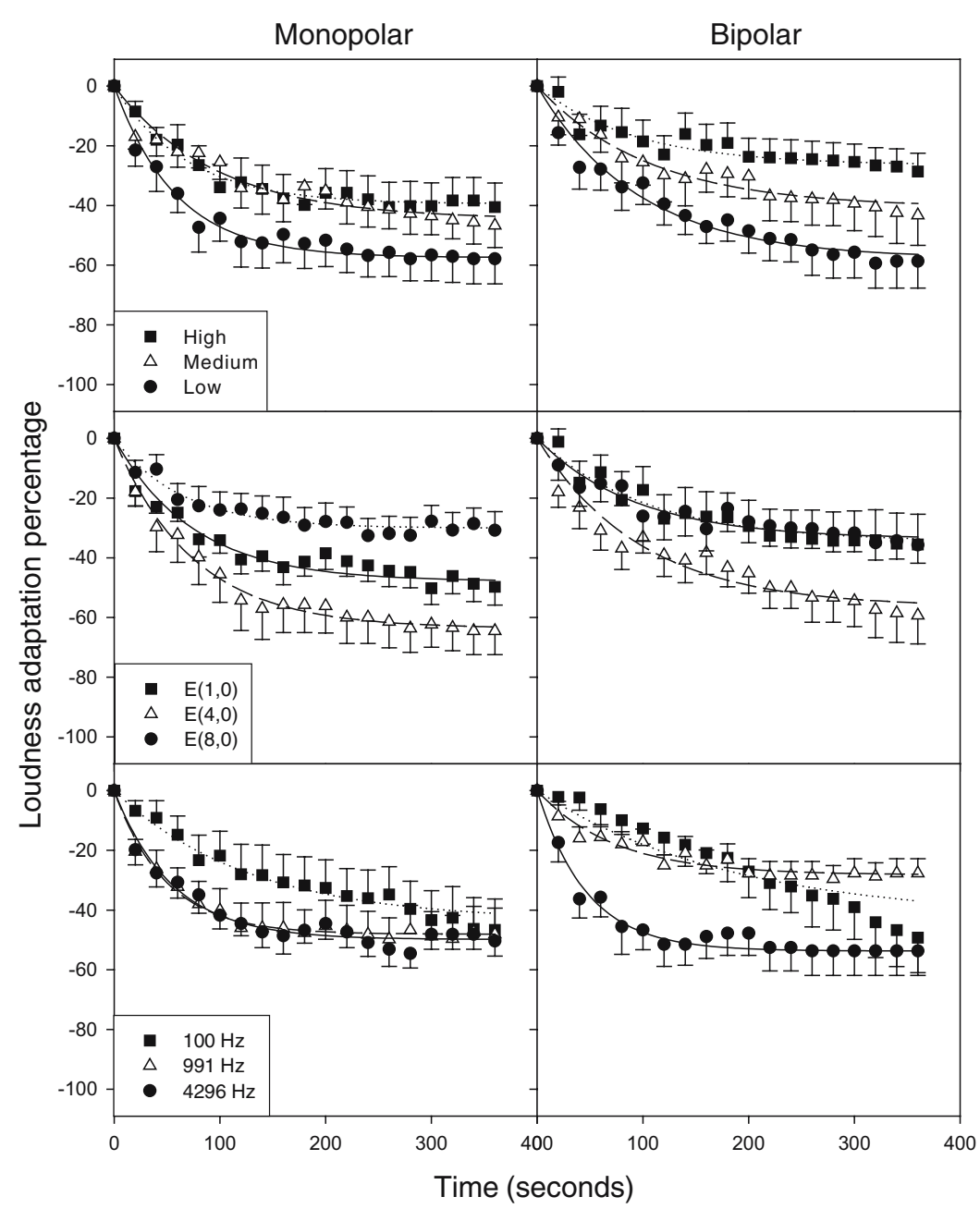

FIG. 5. Average percentage of loudness adaptation (across levels, electrode pair and stimulation frequency) comparison between monopolar and bipolar stimulation mode for all subjects as a function of time. The left column is the monopolar mode and the right column is the bipolar mode. The first row is the average across all levels. The second row is the average across all electrode pairs, and the third row is the average data across all stimulation frequencies. The vertical bars indicate \pm 1 standard error.
The first row shows subject CI-1 as a special case, because the subject could not sustain the perception for all stimulus combinations, reaching total loudness adaptation within $120 \mathrm{~s}$. The second row shows subject CI-2 as another special case because the subject produced essentially no adaptation at the basal electrode. Instead, when the basal electrode was stimulated at medium and high levels, loudness enhancement was observed. The third row shows subject CI-4 who like the remaining two subjects, produced loudness adaptation similar to the normalhearing subjects.

Figure 4 shows individual loudness adaptation data at the $991-\mathrm{Hz}$ rate between monopolar and bipolar stimulation modes in three subjects. The top two subplots are from subject CI-2, who showed no loudness adaptation at the basal electrode in both modes. The four subplots in the middle and bottom show the results from subject CI-3 and CI-5, who have similar loudness adaptation patterns as normal hearing subjects.

To facilitate the examination between the monopolar mode (left column) and the bipolar mode (right column), Fig. 5 compares the level effect by averaging the stimulation rate and position (top row). The electrode position effect is shown by averaging the stimulate level and rate (middle row), and the rate effect is shown by averaging the stimulation level and mode (bottom row). A twotailed, paired $t$-test showed no significant difference between monopolar and bipolar modes $[\mathrm{t}(26)=1.28$, $p>0.05$ ], while the bivariate correlation test showed a significant correlation between the two modes $[R=0.6, p<0.05]$. 


\section{DISCUSSION}

Predicting loudness adaptation in acoustic hearing

In acoustic hearing, significant frequency and level effects were observed on loudness adaptation. Here we used the following second-order polynomial equations to predict the loudness adaptation parameters (i.e., plateaued adaptation percentage, $s$, and time constant, $\tau$ ) using the stimulus parameters (i.e., level, $L$, and frequency, $f$ ):

$$
\begin{gathered}
s=a_{0}+a_{1} f+a_{2} L+a_{3} f^{2}+a_{4} L^{2} \\
\tau=b_{0}+b_{1} f+b_{2} L+b_{3} f^{2}+b_{4} L^{2}
\end{gathered}
$$

Where $a$ and $b$ were fitting parameters that minimized the rms error between the actual and predicted data. Table 3 shows the fitting parameters while Fig. 6 shows the predicted plateaued adaptation percentage ( $Z$-axis in top panel) and time constant (Z-axis in bottom panel) as a function of stimulus frequency and level. Eq. 3 accounted for $96 \%$ variability in the plateaued adaptation percentage data and Eq. 4 for $78 \%$ variability in the time constant data. While the amount of loudness adaptation increases monotonically with increasing frequency and decreasing level, the time constant is a non-monotonic function of both frequency and level. Combining Eqs. (2), (3), and (4), we can theoretically predict loudness adaptation for a pure tone of any given level and frequency in acoustic hearing.

Loudness adaptation in acoustic and electric hearing

At a group level, loudness adaptation averaged across all stimulus conditions was similar in terms of the saturation value and time constant between acoustic hearing ( $s=-40 \%, \tau=82 \mathrm{~s})$ and electric hearing $(s=-54 \%, \tau=99 \mathrm{~s})$. At an individual level, there were apparent differences in loudness adaptation between acoustic and electrical hearing, including a much greater variability in loudness adaptation in cochlear-

\begin{tabular}{|c|c|c|c|c|c|c|c|}
\hline \multicolumn{8}{|c|}{ Coefficients for prediction of loudness adaptation in acoustic hearing } \\
\hline Rate $(\mathrm{Hz})$ & Place & Levels (\% DR) & $\mathrm{Cl}-1$ & $\mathrm{Cl}-2$ & $\mathrm{Cl}-3$ & $\mathrm{Cl}-4$ & $\mathrm{Cl}-5$ \\
\hline \multirow[t]{9}{*}{100} & \multirow[t]{3}{*}{$E(1,0)$} & 10 & $-100 / 116^{*}$ & $-76 / 130$ & $-80 / 223$ & $-100 / 176^{*}$ & $-50 / 1$ \\
\hline & & 50 & $-100 / 168^{*}$ & $-6 / 598$ & $-74 / 200$ & $15 / 69^{* *}$ & $-33 / 4$ \\
\hline & & 90 & $-71 / 225$ & $8 / 77^{* *}$ & $-2 / 267$ & $37 / 94^{* *}$ & $-37 / 59$ \\
\hline & \multirow[t]{3}{*}{$E(4,0)$} & 10 & $-100 / 69^{*}$ & $16 / 23^{* *}$ & $-100 / 57$ & $-25 / 31$ & $-100 / 32 *$ \\
\hline & & 50 & $-100 / 63^{*}$ & $8 / 97^{* *}$ & $-68 / 279$ & $0 / 0^{* * *}$ & $-100 / 62^{*}$ \\
\hline & & 90 & $-100 / 82^{*}$ & $15 / 99 * *$ & $-16 / 169$ & $17 / 4 * *$ & $-50 / 63$ \\
\hline & \multirow[t]{3}{*}{$E(8,0)$} & 10 & $-100 / 46^{*}$ & $20 / 3^{* *}$ & $-80 / 96$ & $-100 / 43^{*}$ & $-50 / 52$ \\
\hline & & 50 & $-100 / 41^{*}$ & $0 / 0 * * *$ & $-63 / 156$ & $-40 / 144$ & $-8 / 0$ \\
\hline & & 90 & $-100 / 95^{*}$ & $0 / 0^{* * *}$ & $-5 / 163$ & $0 / 0 * * *$ & $-7 / 0$ \\
\hline \multirow[t]{9}{*}{991} & \multirow[t]{3}{*}{$E(1,0)$} & 10 & $-100 / 76^{*}$ & $-83 / 44$ & $-66 / 167$ & $-60 / 24$ & $-67 / 53$ \\
\hline & & 50 & $-100 / 116^{*}$ & $-63 / 150$ & $-75 / 271$ & $-50 / 32$ & $-45 / 122$ \\
\hline & & 90 & $-99 / 95$ & $-22 / 104$ & $-88 / 282$ & $-43 / 52$ & $-20 / 26$ \\
\hline & \multirow[t]{3}{*}{$E(4,0)$} & 10 & $-98 / 85$ & $-100 / 85^{*}$ & $-88 / 185$ & $-100 / 157^{*}$ & $-67 / 20$ \\
\hline & & 50 & $-92 / 95$ & $-80 / 26$ & $-92 / 120$ & $-50 / 37$ & $-67 / 78$ \\
\hline & & 90 & $-96 / 61$ & $-93 / 96$ & $-12 / 201$ & $-40 / 41$ & $-80 / 47$ \\
\hline & \multirow[t]{3}{*}{$E(8,0)$} & 10 & $-100 / 6^{*}$ & $-15 / 48$ & $-50 / 227$ & $-100 / 55^{*}$ & $-50 / 1$ \\
\hline & & 50 & $-100 / 72^{*}$ & $11 / 113^{* *}$ & $-100 / 213^{*}$ & $-40 / 31$ & $-25 / 1$ \\
\hline & & 90 & $-100 / 84^{*}$ & $28 / 53^{* *}$ & $-14 / 207$ & $-29 / 48$ & $-40 / 51$ \\
\hline \multirow[t]{9}{*}{4296} & \multirow[t]{3}{*}{$E(1,0)$} & 10 & $-100 / 41^{*}$ & $-67 / 55$ & $-74 / 216$ & $-50 / 31$ & $-50 / 0$ \\
\hline & & 50 & $-100 / 54^{*}$ & $-36 / 21$ & $-68 / 233$ & $-33 / 72$ & $-33 / 3$ \\
\hline & & 90 & $-100 / 72^{*}$ & $-20 / 77$ & $-61 / 240$ & $-20 / 31$ & $-56 / 128$ \\
\hline & \multirow[t]{3}{*}{$E(4,0)$} & 10 & $-100 / 26^{*}$ & $-75 / 119$ & $-36 / 227$ & $-75 / 92$ & $-67 / 20$ \\
\hline & & 50 & $-100 / 133^{*}$ & $-28 / 61$ & $-28 / 248$ & $-50 / 85$ & $-55 / 45$ \\
\hline & & 90 & $-100 / 236^{*}$ & $-54 / 125$ & $-14 / 158$ & $-40 / 81$ & $-50 / 85$ \\
\hline & \multirow[t]{3}{*}{$E(8,0)$} & 10 & $-100 / 31^{*}$ & $20 / 0^{* *}$ & $-65 / 198$ & $-76 / 74$ & $-67 / 45$ \\
\hline & & 50 & $-100 / 94^{*}$ & $7 / 720 * *$ & $-27 / 251$ & $-50 / 59$ & $-50 / 32$ \\
\hline & & 90 & $-100 / 143^{*}$ & $38 / 95^{* *}$ & $0 / 0 * * *$ & $-29 / 52$ & $-52 / 54$ \\
\hline
\end{tabular}

TABLE 3

*Total adaptation.

**Loudness enhancement.

***No adaptation. 


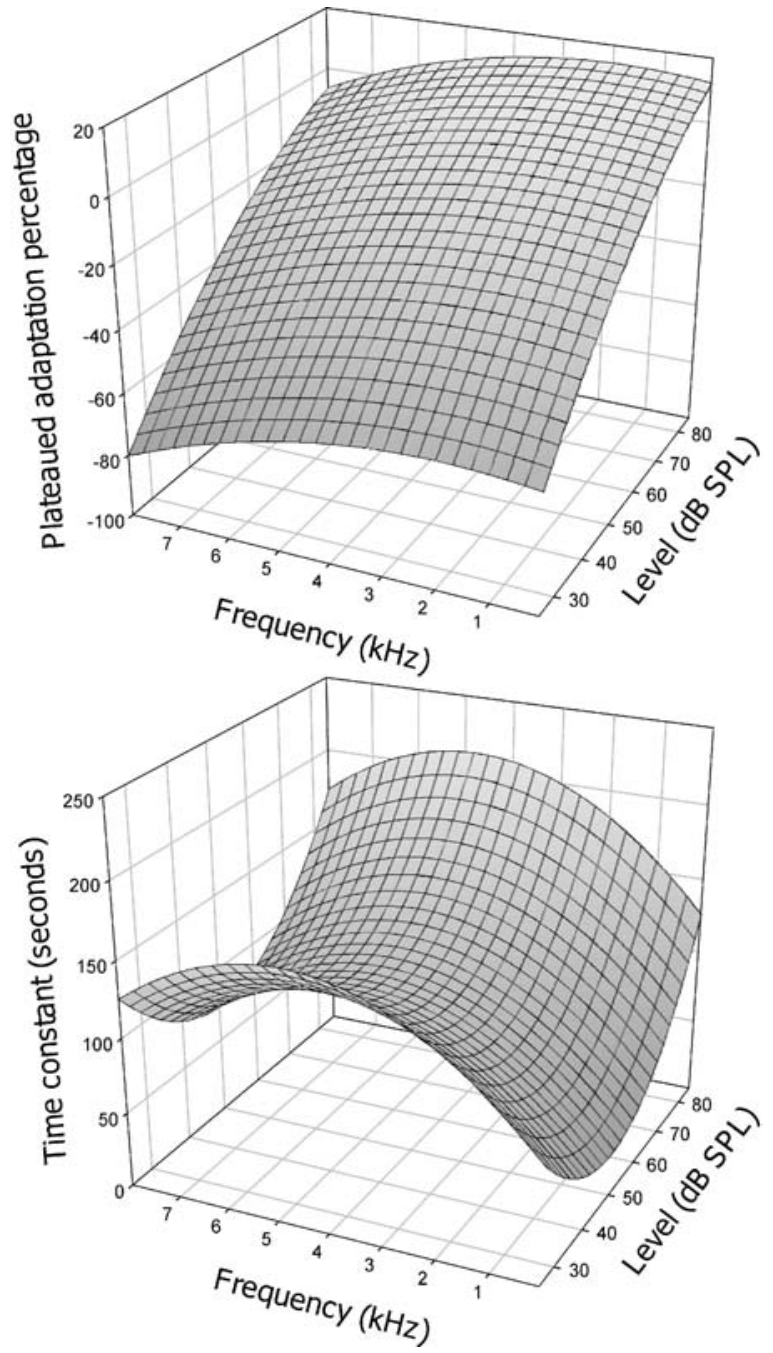

FIG. 6. Predicted plateaued adaptation percentage (top panel) and time constant (bottom panel) by using quantitative model of loudness adaptation in acoustic hearing. For details see Eqs. 3 and 4 in the text.

implant subjects. Different from electric hearing, small but significant loudness enhancement was observed at $90 \mathrm{~dB}$ SPL in all normal-hearing subjects; total loudness adaptation was never observed in any normal-hearing subjects.

The greater variability might be due to various etiologies and nerve survival patterns in the cochlearimplant subjects. The loudness adaptation difference between acoustic and electric hearing might also be due to different loudness growth between different stimulus configurations. For example, equal-loudness contours indicate that the loudness at different levels $(30,60$, and $90 \mathrm{~dB})$ varies significantly for different frequencies in acoustic hearing (Fletcher and Munson 1933). Similarly, the loudness at three different levels $(10,50$, and $90 \%$ of the electric dynamic range) also varies significantly at different stimulation rates, electrode locations, or stimulation mode (Shannon
1985; Zeng and Shannon 1994; Chatterjee 1999; Chatterjee et al. 2000).

\section{Loudness adaptation mechanisms}

The present results were not fully compatible with either the restricted excitation hypothesis or the neural adaptation hypothesis. First, psychophysical and physiological studies have demonstrated that the monopolar stimulation produces a wider excitation pattern than the bipolar stimulation mode and that basal electrodes produce more spread towards apical and possibly wider spread of excitation than apical electrodes (van den Honert and Stypulkowski 1987; Lim et al. 1989; Chatterjee and Shannon 1998; Kral et al. 1998; Bierer and Middlebrooks 2002, 2004; Middlebrooks and Bierer 2002; Miller et al. 2003; Middlebrooks 2004). The restricted excitation hypothesis would predict greater degrees of loudness adaptation with the bipolar mode than the monopolar mode and with basal electrodes than apical electrodes. The present results showed that neither electrode position nor stimulation mode was a significant factor in determining loudness adaptation. Second, the neural adaptation model would predict that loudness adaptation increases with stimulate rate and level. However, the present results showed that the low-rate stimulation $(100 \mathrm{~Hz})$ produced as much loudness adaptation as the highrate $(991$ and $4,296 \mathrm{~Hz})$ stimulation [for plateaued adaptation percentage: $\mathrm{F}(2,8)=3.5, p>0.05$; for time constant: $\mathrm{F}(2,8)=1.0, p>0.05]$. To parsimoniously explain loudness adaptation in acoustic and electric hearing, we propose a loudness adaptation mechanism that contains a central feedback loop with the gain dependent on the peripheral neural activity. At low stimulus levels, the low total spike count produced a negative gain, decreasing the perceived loudness so that the listener can ignore the stimulus. As the level increases, the gain becomes one so that the perceived loudness is constant for maintaining comfortable and stable listening. As the level further increases, a positive gain is produced to induce loudness enhancement, alerting the listener to avoid potential damage to the auditory system. Because loudness enhancement is observed only $9 \%$ of the time and mostly in one subject (CI-2) in the present data, this positive gain may be applicable to only acoustic hearing.

\section{Implications for application}

High-rate conditioners have been shown to produce pseudo-spontaneous activity in electrically stimulated auditory nerve fibers (Rubinstein et al. 1999). To apply the high-rate conditioner to actual implant 
users, one has to assume that total loudness adaptation is obtained so that the high-rate condition would not interfere with other information-carrying stimuli. While previous studies showed that total loudness adaptation appeared to be reached in most tested implant users (Hong et al. 2003), the present result showed that there is large individual variability. As a matter of fact, four out of five subjects (CI-2, 3, 4 and 5) did not produce total loudness adaptation in most of the stimulus conditions (see Fig. 3). In CI-1, total loudness adaptation was reached for all stimulation rates and levels, suggesting a possible etiology of neuropathy in this subject. In CI-3, 4 and 5, total adaptation was reached only at low rates and low levels, suggesting a possible central mechanism rather than a neural adaptation mechanism. In CI-2, no total adaptation was observed under any conditions. The present results suggest that potential use of conditioners in cochlear implants should take this individual variability into account.

\section{SUMMARY}

Loudness adaptation was systematically measured in five normal-hearing and five cochlear-implant subjects. Successive magnitude estimation was used to quantify loudness adaptation over a 367 -s period. The following conclusions were reached:

1. Consistent with previous studies in acoustic stimulation, the present study found a significant effect of stimulus level and frequency on loudness adaptation: The lower the stimulus level and the higher the stimulus frequency, the greater the adaptation.

2. Different from previous studies in acoustic stimulation, the present study observed a small but significant loudness enhancement effect at the highest stimulus level (90 dB SPL) for all stimulus frequencies.

3. Similar to acoustic hearing, the present study found a significant effect of stimulus level on loudness adaptation in electric hearing. However, loudness adaptation in electric hearing was not significantly dependent on stimulation rate, place, and mode.

4. Different from previous studies in electric hearing, the present study observed a great deal of individual variability in the implant subjects' ability to reach total loudness adaptation, implying that potential use of high-rate conditioners in cochlear implants should take this variability into account.

5 . The present result was not consistent with the existing models of loudness adaptation, rather it suggested a possible central feedback mechanism.

\section{ACKNOWLEDGMENTS}

This research was supported by the National Institutes of Health (2-RO1 DC002267). We would like to thank all subjects for their participation in this research. Abby Copeland, Jay Rubinstein, Eric D. Young and two anonymous reviewers provided helpful comments on the manuscript.

\section{REFERENCES}

Bierer JA, Middlebrooks JC. Auditory cortical images of cochlearimplant stimuli: dependence on electrode configuration. J. Neurophysiol. 87:478-492, 2002.

Bierer JA, Middlebrooks JC. Cortical responses to cochlear implant stimulation: channel interactions. J. Assoc. Res. Otolaryngol. 5:32-48, 2004.

Brimacombe JA, EIsenberg LS. Tone decay in subjects with the single-channel cochlear implant. Audiology 23:321-332, 1984.

Bruce IC, Irlicht LS, White MW, O’Leary SJ, Dynes S, Javel E, Clark GM. A stochastic model of the electrically stimulated auditory nerve: pulse-train response. IEEE Trans. Biomed. Eng. 46:630$637,1999 \mathrm{a}$.

Bruce IC, White MW, Irlicht LS, O'Leary SJ, Dynes S, Javel E, Clark GM. A stochastic model of the electrically stimulated auditory nerve: single-pulse response. IEEE Trans. Biomed. Eng. 46:617629, 1999b.

CARHART R. Clinical determination of abnormal auditory adaptation. AMA Arch. Otolaryngol. 65:32-39, 1957.

CARNEY LH. Spatiotemporal encoding of sound level: models for normal encoding and recruitment of loudness. Hear Res. 76:31-44, 1994 .

Chatterjee M. Effects of stimulation mode on threshold and loudness growth in multielectrode cochlear implants. J. Acoust. Soc. Am. 105:850-860, 1999.

Chatterjee M, Shannon RV. Forward masked excitation patterns in multielectrode electrical stimulation. J. Acoust. Soc. Am. 103:2565-2572, 1998.

Chatterjee M, Fu QJ, Shannon RV. Effects of phase duration and electrode separation on loudness growth in cochlear implant listeners. J. Acoust. Soc. Am. 107:1637-1644, 2000.

Fletcher H. Auditory patterns. Rev. Modern Phys. 12:47-66, 1940.

Fletcher H, Munson WA. Loudness, its definition, measurement and calculation. J. Acoust. Soc. Am. 5:82-108, 1933.

Fowler E. A method for the early detection of otosclerosis. Arch. Otolaryngol. 24:731-741, 1936.

GREEN DM. Stimulus selection in adaptive psychophysical procedures. J. Acoust. Soc. Am. 87:2662-2674, 1990.

GREEN DS. Tone decay. Handbook of clinical audiology. J Katz. New York, Williams \& Wilkins, 1985.

Hellman R, Miskiewicz A, Scharf B. Loudness adaptation and excitation patterns: effects of frequency and level. J. Acoust. Soc. Am. 101:2176-2185, 1997.

Hong RS, Rubinstein JT, Wehner D, Horn D. Dynamic range enhancement for cochlear implants. Otol. Neurotol. 24:590595, 2003.

Hood JD. Studies in auditory fatigue and adaptation. Acta Otolaryngol., Suppl. 92:1-57, 1950.

Hoop JD. Auditory fatigue and adaptation in the differential diagnosis of end-organ disease. Ann. Otol. Rhinol. Laryngol. 64:507-518, 1955

Huss M, Moore BC. Tone decay for hearing-impaired listeners with and without dead regions in the cochlea. J. Acoust. Soc. Am. 114:3283-3294, 2003. 
ISO. Method for calculating loudness level. International Standardization Organization R532, 1966.

JAVEL E. Long-term adaptation in cat auditory-nerve fiber responses. J. Acoust. Soc. Am. 99:1040-1052, 1996.

Kral A, Hartmann R, Mortazavi D, Klinke R. Spatial resolution of cochlear implants: the electrical field and excitation of auditory afferents. Hear Res. 121:11-28, 1998.

Lim HH, Tong YC, Clark GM. Forward masking patterns produced by intracochlear electrical stimulation of one and two electrode pairs in the human cochlea. J. Acoust. Soc. Am. 86:971-980, 1989.

Middlebrooks JC. Effects of cochlear-implant pulse rate and interchannel timing on channel interactions and thresholds. J. Acoust. Soc. Am. 116:452-468, 2004.

Middlebrooks JC, Bierer JA. Auditory cortical images of cochlearimplant stimuli: coding of stimulus channel and current level. J. Neurophysiol. 87:493-507, 2002.

Miller CA, Abbas PJ, Nourski KV, Hu N, Robinson BK. Electrode configuration influences action potential initiation site and ensemble stochastic response properties. Hear Res. 175:200214, 2003.

Miskiewicz A, Hellman R. Frequency and temporal effects in loudness adaptation. In: 15th International Congress on Acoustics 2, 1995.

Miskiewicz A, Scharf B, Hellman R, Meiselman C. Loudness adaptation at high frequencies. J. Acoust. Soc. Am. 94:12811286, 1993.

Moore BC. Loudness perception and intensity coding. Hearing, 1995.

Moore BC, Glasberg BR. A revised model of loudness perception applied to cochlear hearing loss. Hear Res. 188:70-88, 2004.

Pelizzone M. Very high stimulation rates for cochlear implants. In: 4th European Symposium on Pediatric Cochlear Implantation. Hertogenbosch, The Netherlands, 1998.

Relkin EM, Doucet JR. Is loudness simply proportional to the auditory nerve spike count? J. Acoust. Soc. Am. 101:2735-2740, 1997.

Robinson DW, DADSON RS. A redetermination of the equal-loudness relations for the pure tones. Br. J. Appl. Phys. 7:166-181, 1956.
Rubinstein JT, Wilson BS, Finley CC, Abbas PJ. Pseudospontaneous activity: stochastic independence of auditory nerve fibers with electrical stimulation. Hear Res. 127:108-118, 1999.

Rubinstein JT, Tyler RS, Johnson A, Brown CJ. Electrical suppression of tinnitus with high-rate pulse trains. Otol. Neurotol. 24:478-485, 2003.

Scharf B. Loudness adaptation. Hearing research and theory. JV Tobias, ED Schubert. 2, 1983.

Schlauch RS, Digiovanni JJ, Ries DT. Basilar membrane nonlinearity and loudness. J. Acoust. Soc. Am. 103:2010-2020, 1998.

Schlauch RS, Ries DT, Digiovanni JJ. Duration discrimination and subjective duration for ramped and damped sounds. J. Acoust. Soc. Am. 109:2880-2887, 2001.

Sennaroglu G, Sennaroglu L, Yucel Ee, Belgin E. Cochlear implants: is there any relationship between stimulation rates and adaptation. Otol. Neurotol. 22:634-636, 2001.

SHANNon RV. Threshold and loudness functions for pulsatile stimulation of cochlear implants. Hear Res. 18:135-143, 1985.

Stevens SS. Neural events and the psychophysical law. Science 170:1043-1050, 1970.

VAN DEN Honert C, STypulkowski PH. Temporal response patterns of single auditory nerve fibers elicited by periodic electrical stimuli. Hear Res. 29:207-222, 1987.

Wable J, Frachet B, Gallego S. Tone decay at threshold with auditory electrical stimulation in digisonic cochlear implantees. Audiology 40:265-271, 2001.

Wilson BS, Finley CC, Lawson DT, Wolford RD, Zerbi M. Design and evaluation of a continuous interleaved sampling (CIS) processing strategy for multichannel cochlear implants. J. Rehabil. Res. Dev. 30:110-116, 1993.

Winter IM, Robertson D, Yates GK. Diversity of characteristic frequency rate-intensity functions in guinea pig auditory nerve fibres. Hear Res. 45:191-202, 1990.

Zeng FG, Shannon RV. Loudness-coding mechanisms inferred from electric stimulation of the human auditory system. Science 264:564-566, 1994.

Zwicker E, Scharf B. A model of loudness summation. Psychol. Rev. 72:3-26, 1965. 